\title{
Effects of Structure on the Tribological Properties of Organic Self-Assembled Molecular Layers
}

\author{
Miki Nakano* and Koji Miyake \\ Advanced Manufacturing Research Institute, National Institute of Advanced Industrial Science and Technology (AIST) \\ 1-2-1 Namiki, Tsukuba-shi, Ibaraki 305-8564, Japan \\ *Corresponding author: mi.nakano@aist.go.jp
}

( Manuscript received 28 March 2013; accepted 25 November 2013; published 31 December 2013 )

\begin{abstract}
The tribological properties of self-assembled monolayers (SAMs) on Au were investigated with a focus on the effects of the structure and end groups of the molecules upon the frictional properties of the modified surfaces of the SAMs. Three kinds of organothiols were used: $\mathrm{HS}-\left(\mathrm{CH}_{2}\right)_{11}-\left(\mathrm{O}-\mathrm{CH}_{2}-\mathrm{CH}_{2}\right)_{3}-\mathrm{OCH}_{3}$ : EG3OMe, $\mathrm{CH}_{2}-\left(\mathrm{CH}_{2}\right)_{15}-\mathrm{SH}$ : HDT, and $\mathrm{OH}-\left(\mathrm{CH}_{2}\right)_{16}-\mathrm{SH}$ : HDTOH. The SAM surfaces were characterized by contact angle measurements of pure water, X-ray photoelectron spectroscopy, and infrared reflection absorption spectroscopy. The frictional properties were examined using a pin-on-plate tribometer. The friction coefficients of the SAM surfaces were similar to the bare Au surface in the high normal load region. In the low normal load region, HDT SAMs exhibited lower friction coefficients than HDTOH SAMs. The end groups affected the friction coefficients for the same chain structures. The friction coefficients of the EG3OMe SAMs were lower than those of HDT SAMs. The HDT SAMs were thought to have well-organized structures. On the other hand, the EG3OMe SAMs probably had more flexible structures than the HDT SAMs because of their ethylene glycol chains, indicating that the chain structures as well as the end groups affected the friction coefficients of the SAMs.
\end{abstract}

Keywords: SAM, friction, ethylene glycol, XPS, FT-IR

\section{Introduction}

The tribological properties of organic thin films like self-assembled monolayers (SAMs) have been investigated [1-7] using both nano-scale tribometers such as friction force microscopy and macro-scale tribometers because such films exhibit lower friction coefficients than bare substrates. SAMs are expected to be used especially as functional ultra-thin molecular layers in such applications as lubrication and organic electronic devices because of their ease of preparation. SAMs can be applied to various kinds of surfaces using molecules with different head groups, such as thiol, silanes, multiple $\mathrm{C}-\mathrm{C}$ bonds, and acids. The tribological properties of SAMs are affected by several factors which have been investigated by many researchers and include head groups, chain lengths, chain structures, chemical bonds inside the chains, and end groups.

Early studies of the tribological properties of SAMs have mainly used organosulfurs on $\mathrm{Au}$ and organosilanes on silicon ( $\mathrm{Si}$ ). Recently, Si has become one of the most popular substrates in use because of the focus on microelectromechanical systems (MEMS). The possibility of using $\mathrm{SAMs}$ on $\mathrm{Si}$ as lubricants for
MEMS has been reported [4,5,8-11] using atomic force microscopy (AFM) techniques because of their small thickness and chemical bonding with the substrates. Furthermore, it is easy to control the surface properties of MEMS, such as hydrophilicity, using SAMs, as well as to change the thickness of the SAMs. In previous studies on the tribological properties of SAMs on $\mathrm{Si}$ $[4,5]$, mainly organosilane SAMs have been used. It has been reported that organosilane SAMs reduced the friction coefficients, stiction and wear. Furthermore, tests using macro-scale tribometers, such as traditional pin-on-disk or pin-on-plate tribometers, have been conducted. These studies have also demonstrated that SAMs on $\mathrm{Si}$ had the potential for application as lubricants [7].

Basic studies on the tribological properties of SAMs have been also performed using organosulfur on $\mathrm{Au}$ [12-15] for a number of reasons. One reason is that there are many kinds of organosulfur compounds which can easily form SAMs. Also, SAMs on Au substrates are suitable for investigating the influence of molecules (molecular length, functional group, chain structure) upon the tribological behavior. Another reason is that the SAM formation process on $\mathrm{Au}$ substrates is easier 
than that on other substrates such as silicon made from organosilianol compounds. For example, the authors have investigated the tribological behavior of organosulfur molecule SAMs on Au using a pin-on-plate method [13-15]. In our previous study, we found that the friction coefficients were dependent on the alkyl chain length and that even a monolayer film was sufficiently durable against friction, although the friction coefficients were slightly high at 0.2 to 0.4 . As such, a decrease in the friction coefficient of alkanethiol monolayers is necessary for SAMs to be effectively used as lubricants. In addition, further measurements to increase their durability against friction are required. We also investigated the frictional properties of terphenyl methane thiol (TP1) SAMs [14]. Oligophenylene SAMs containing phenyl rings are also expected to be good surface coating materials because they have been predicted to be rigid [16]. Although the friction coefficients of terphenyl methane thiol SAMs measured by friction force microscopy were higher than those of soft octadecanethiol (ODT) SAMs, when measured by the pin-on-plate method, both of these SAMs exhibited almost the same friction coefficients. With pin-on-plate measurements, the deformation of the substrates arising from the higher sliding speed may affect the friction behavior. Furthermore, X-ray photoelectron spectroscopy (XPS) measurements have indicated that TP1 SAMs have higher durability against friction than ODT SAMs.

The formation of overlayers on a monolayer seems to be effective for increasing the durability and decreasing the friction coefficient. It has been reported that the formation of a new overlayer on a monolayer can decrease the friction caused by polymers, as in the case of perfluoropolyether (PFPE) [17]. The authors have also reported on the tribological properties of self-assembled double layers on Au. The self-assembled double layers had effect of lowering friction. However, their durability against friction was inadequate for practical use. Another candidate is SAMs with molecules having double units. Recently, oligo (ethylene glycol) (OEG)-terminated alkanethiol SAMs have been investigated for the purpose of protein resistance. These molecules have two units of ethylene glycol and alkyl chains. Thus, OEG-terminated alkanethiol SAMs have the potential for use as a lubricant film.

In this present study, we prepared OEG-terminated alkanethiol, alkanethiol, and hydroxyl terminated alkanethiol SAMs on Au. The SAM surfaces were characterized using contact angle measurements of pure water, XPS, and infrared reflection-absorption spectroscopy (IRRAS). The tribological properties were examined using a macro-scale tribotester to clarify the effects of the end groups and the chain structures of the molecules on the friction properties.

\section{Experimental Methods}

\subsection{SAM preparation}

The Au substrate was prepared by sputtering $\mathrm{Cr}$ and $\mathrm{Au}$ on $\mathrm{Si}\left(\begin{array}{lll}1 & 1 & 1\end{array}\right)$. After a $\mathrm{Cr}$ layer was deposited to improve adhesion between the Au film and Si substrate, the $\mathrm{Au}$ film was formed. The thickness of the $\mathrm{Au}$ and $\mathrm{Cr}$ films was $150 \mathrm{~nm}$ and $10 \mathrm{~nm}$, respectively. The $\mathrm{Au}$ substrates were cleaned by ultraviolet light (UV) and ozone treatment for $30 \mathrm{~min}$ using a UV/ozone cleaner (Nippon Lazar \& Electronics Lab.) to oxidize the $\mathrm{Au}$ surface. The substrates were rinsed with a pure ethanol solution for $30 \mathrm{~min}$ to remove the oxide [18-20]. Self-assembled monolayers were prepared on each $\mathrm{Au}$ substrate in a glass beaker at room temperature. The cleaned Au substrate was exposed to a solution of $1 \mathrm{mM}$ of organothiol in ethanol for $24 \mathrm{~h}$. The organothiols used in this study are listed in Table 1 and are HS- $\left(\mathrm{CH}_{2}\right)_{11}-\left(\mathrm{O}-\mathrm{CH}_{2}-\mathrm{CH}_{2}\right)_{3}-\mathrm{OCH}_{3}$ : EG3OMe, $\mathrm{CH}_{2}-\left(\mathrm{CH}_{2}\right)_{15}-\mathrm{SH}$ : HDT and $\mathrm{OH}-\left(\mathrm{CH}_{2}\right)_{16}-\mathrm{SH}$ : HDTOH. These three organothiols with different end groups of the methyl group and hydroxyl group, and different chain structures of alkanethiol chain and OEG-terminated alkanethiol were used to examine the effects of the end groups and the chain structures of the molecules on the friction properties. The HDT, HDTOH, and EG3OMe were purchased from Tokyo Chemical Industry Co., Ltd., Dojindo Molecular Technologies, Inc., and ProChimia Surfaces. Sp.z o.o., respectively, and were used as received. While being exposed to organothiol solution, organothiols (R-S-H) adsorbed on Au surface, gold thiolates (R-S-Au) formed, and SAMs formed. After removal from the solution, the Au substrates were rinsed with pure ethanol to remove the physisorbed multilayer of organothiol.

\subsection{Characterization of the SAM surfaces}

The SAM surfaces were characterized by contact angle measurements, XPS analysis, and IR-RAS analysis. Static water contact angle measurements were performed using deionized water (MilliQ). Four measurements were made on each sample.

Table 1 Abbreviations and structural formula of molecules

\begin{tabular}{c|c|c}
\hline Abbreviations & Structural formula & End groups \\
\hline HDT & $\mathrm{SH}-\left(\mathrm{CH}_{2}\right)_{15}-\mathrm{CH}_{3}$ & $-\mathrm{CH}_{3}$ \\
\hline $\mathrm{HDTOH}$ & $\mathrm{SH}-\left(\mathrm{CH}_{2}\right)_{16}-\mathrm{OH}$ & $-\mathrm{OH}$ \\
\hline EG3OMe & $\mathrm{HS}-\left(\mathrm{CH}_{2}\right)_{11}-\left(\mathrm{O}-\mathrm{CH}_{2}-\mathrm{CH}_{2}\right)_{3}-\mathrm{OCH}_{3}$ & $-\mathrm{OCH}_{3}$ \\
\hline
\end{tabular}


XPS spectra were recorded using a Theta Probe XPS system (Thermo Fisher Scientific K.K.) with a monochromatic Al-Ka X-ray source $(1486.6 \mathrm{eV})$. The base pressure of the XPS system was less than $3 \times 10^{-7}$ $\mathrm{Pa}$. The X-ray power, pass energy of the analyzer, and take-off angle of photoelectrons were set at $10 \mathrm{~W}, 100$ $\mathrm{eV}$, and $90 \mathrm{deg}$., respectively. The binding energies were corrected by using the $\mathrm{Au}\left(4 \mathrm{f}_{7 / 2}\right)$ peak $(84.0 \mathrm{eV})$ as an energy standard. The peak area was normalized by a sensitivity factor for each element at 1.00, 1.68, and 17.1 for $\mathrm{C}(1 \mathrm{~s}), \mathrm{S}(2 \mathrm{p})$, and $\mathrm{Au}(4 \mathrm{f})$, respectively [21].

Infrared reflection-absorption spectra were recorded on an FT-IR system (Mattson Infinity Gold) equipped with a grazing angle $\left(80^{\circ}\right)$ infrared reflection accessory and a liquid nitrogen cooled mercury cadmium telluride (MCT) detector. A spectrum of a bare Au surface was used as a reference.

\subsection{Friction measurements}

The frictional properties were examined using an in-house developed pin-on-plate tribometer (cf. Fig. 1). Linear reciprocating friction tests were performed. The sliding speed was varied in sinusoidal and the average speed was 1.0 to $10.0 \mathrm{~mm} / \mathrm{s}(0.1$ to $0.1 \mathrm{~Hz})$, and the stroke was $5 \mathrm{~mm}$. The normal load was varied from 9.8 to $98 \mathrm{mN}$ and the mean Hertzian contact pressure was estimated to be approximately 53 to $116 \mathrm{MPa}$. The surface roughness of the glass pin and Au substrate were $0.019 \mu \mathrm{m} R a$ and $0.003 \mu \mathrm{m} \mathrm{Ra}$, respectively. The local pressure at the tip asperities is higher than 53 to 116 $\mathrm{MPa}$ because the surface roughness of the glass pin is higher than that of the Au surface. The friction coefficients were averaged over a sliding distance of 0.3 $\mathrm{m}$. The experimental temperature was $298 \mathrm{~K}$, and the humidity was kept at 30 to $40 \%$ by a flow of dry nitrogen. The pins were made of borosilicate glass (Pyrex), with a diameter of $3 \mathrm{~mm}$ and a length of $20 \mathrm{~mm}$. The pins were cleaned in acetone, ethanol, and deionized water, and then dried at $393 \mathrm{~K}$ for $10 \mathrm{~min}$. After cleaning, the ends of the pins were flame-annealed to produce a smooth surface.

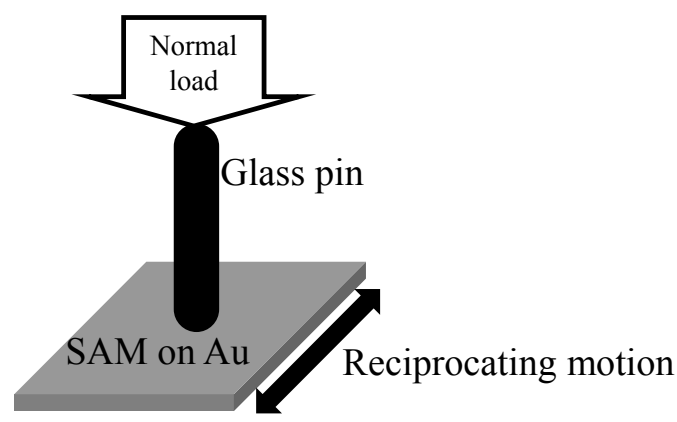

Fig. 1 Schematic diagram of pin-on-plate tribometer
Table 2 Contact angle using pure water measured on the HDT, HDTOH and EG3OMe SAMs

\begin{tabular}{c|c}
\hline Abbreviations & Contact angle (water) \\
\hline HDT & $105.2^{\circ} \pm 0.9^{\circ}$ \\
\hline HDTOH & $19.6^{\circ} \pm 1.7^{\circ}$ \\
\hline EG3OMe & $52.9^{\circ} \pm 0.9^{\circ}$ \\
\hline
\end{tabular}

\section{Results and Discussions}

\subsection{Contact angle measurements}

The effects of surface wettability and surface energy upon the friction properties were investigated. The results of contact angle measurements using pure water are shown in Table 2, and the numbers are average values of four results. The HDT and HDTOH SAMs were hydrophobic and hydrophilic, respectively, because of the effects of their end groups. Although both the EG3OMe and HDT SAMs have the methyl groups as the end group, the contact angle of the HDT SAM is higher than that of the EG3OMe SAM. The reason for the lower contact angle of the EG3OMe is the exposure of the ethylene glycol (EG) units which contain oxygen atoms at the outer surface [22].

\subsection{XPS analysis}

XPS measurements were performed on all SAMs. Figure 2 shows the XPS spectra of the HDT (a), HDTOH (b), and EG3OMe SAMs in the C(1s) region. For both the HDT and HDTOH SAMs, a strong peak was observed in the $\mathrm{C}(1 \mathrm{~s})$ region at approximately $285.0 \mathrm{eV}$, which was assigned to the aliphatic chains. For the HDTOH SAM, a shoulder peak was observed at approximately $286.8 \mathrm{eV}$, which was assigned to the C-O-H bond. On the other hand, for the EG3OMe SAM, two peaks were observed at approximately $284.7 \mathrm{eV}$ and $286.6 \mathrm{eV}$, which were assigned to the aliphatic chains and the ether carbons, respectively [23]. The XPS spectra showed that the EG3OMe SAM had two units which were the aliphatic chains and ethylene glycol units. We estimated the XPS peak area ratio of

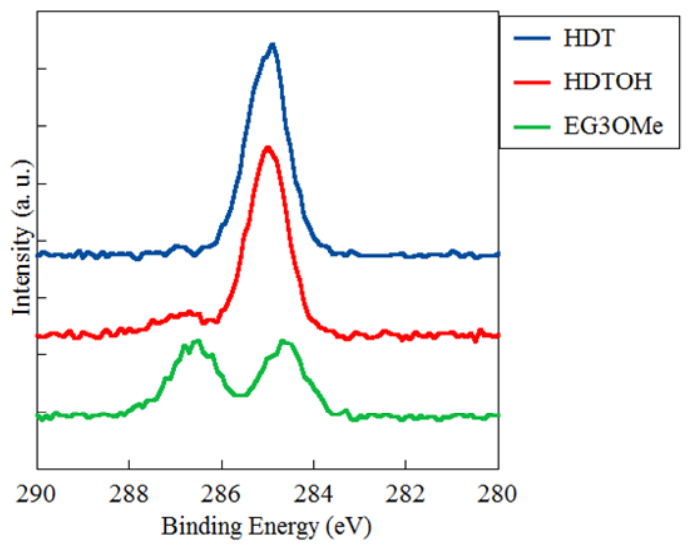

Fig. 2 XPS spectra in the $\mathrm{C}(1 \mathrm{~s})$ region of the HDT, HDTOH and EG3OMe SAMs 


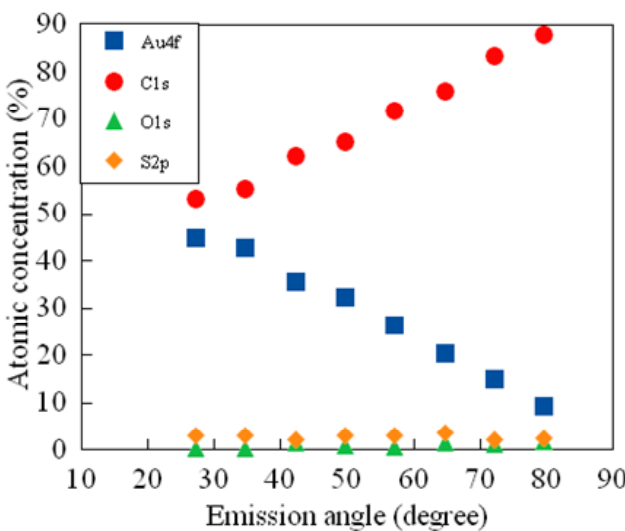

(a) HDT

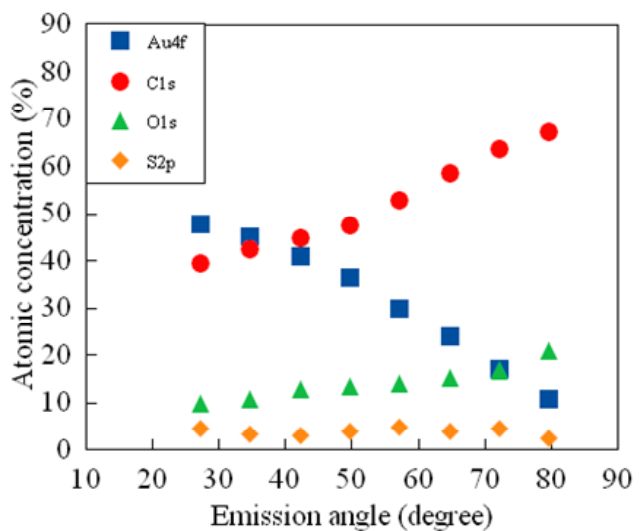

(b) EG3OMe

Fig. 3 Atomic concentration of the HDT (a) and EG3OMe (b) SAMs as a function of emission angle using angle-resolved XPS spectra

$\mathrm{C}(1 \mathrm{~s}) / \mathrm{Au}(4 \mathrm{f})$ to examine the packing density of the SAMs. The XPS peak area ratio of $\mathrm{C}(1 \mathrm{~s}) / \mathrm{Au}(4 \mathrm{f})$ on the HDT, HDTOH and EG3OMe SAMs were $1.60,2.42$ and 1.71 , respectively. The number of carbon atoms in the HDT, HDTOH and EG3OMe molecules were 16, 16, and 18 , respectively. As such, the packing density of the HDT SAM was close to that of the EG3OMe SAM in this study. The high XPS peak area ratio of $\mathrm{C}(1 \mathrm{~s}) / \mathrm{Au}(4 \mathrm{f})$ measured on the HDTOH SAM could be due to the physisorbed HDTOH molecules. We think that the physisorbed molecules adsorbed weakly and desorbed during the friction tests. We also performed angle-resolved XPS measurements. Figure 3 shows the atomic concentration of the HDT (a) and EG3OMe (b) SAMs as a function of the emission angle. The high and low emission angle regions show the surface and bulk regions, respectively. Both for the HDT and EG3OMe SAMs, the atomic concentration of $\mathrm{C}$ was high and that of $\mathrm{Au}$ was low in the surface region. Moreover, for the EG3OMe SAM, the atomic concentration of $\mathrm{O}$ was high

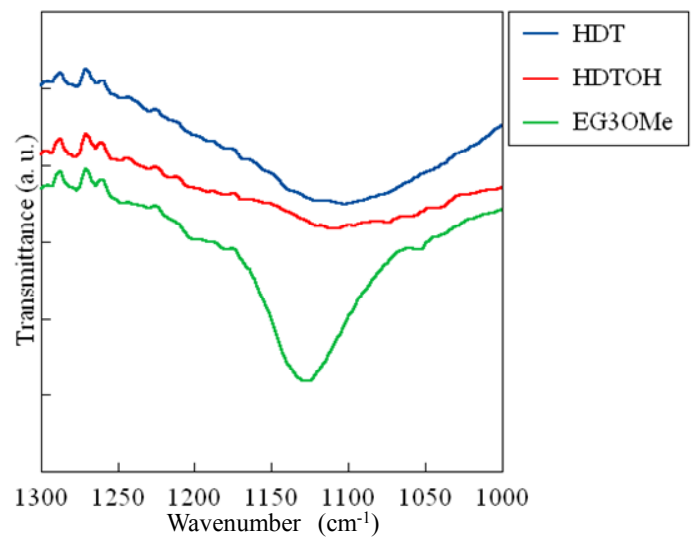

Fig. 4 The fingerprint region of the IRRAS spectra for the HDT, HDTOH, and EG3OMe SAMs in the surface region, indicating that EG units of the EG3OMe SAM were present on the surface.

\subsection{FT-IR analysis}

The surfaces were characterized by FT-IR spectroscopy to further verify the formation of the SAMs on $\mathrm{Au}$. Figure 4 shows the fingerprint region of the IRRAS spectra for the HDT (a), HDTOH (b), and EG3OMe (c) SAMs. The EG3OMe spectrum shows a strong characteristic peak at $1126 \mathrm{~cm}^{-1}$, which was assigned to the C-O-C stretching mode [24]. On the other hand, only a broad peak was observed for the HDT and HDTOH SAMs of the fingerprint region. Figure 5 shows the $\mathrm{CH}$ stretching region of the IRRAS spectra for the HDT (a), HDTOH (b), and EG3OMe (c) SAMs. The HDT spectrum shows four peaks at $2850 \mathrm{~cm}^{-1}, 2877 \mathrm{~cm}^{-1}$, $2920 \mathrm{~cm}^{-1}$, and $2962 \mathrm{~cm}^{-1}$, which were assigned to the $\mathrm{CH}_{2}$ symmetrical stretching mode, $\mathrm{CH}_{3}$ symmetrical stretching mode, $\mathrm{CH}_{2}$ antisymmetric stretching mode, and $\mathrm{CH}_{3}$ antisymmetric stretching mode, respectively

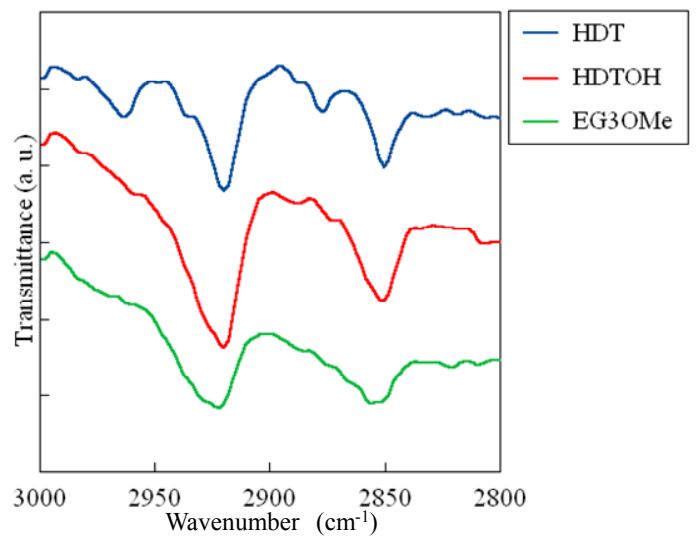

Fig. 5 The $\mathrm{CH}_{2}$ stretching region of the IRRAS spectra for the HDT, HDTOH, and EG3OMe SAMs 


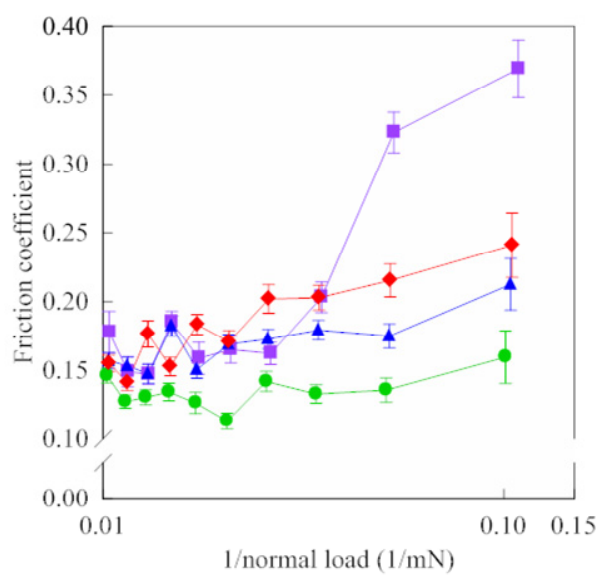

(a) $1 \mathrm{~mm} / \mathrm{sec}$

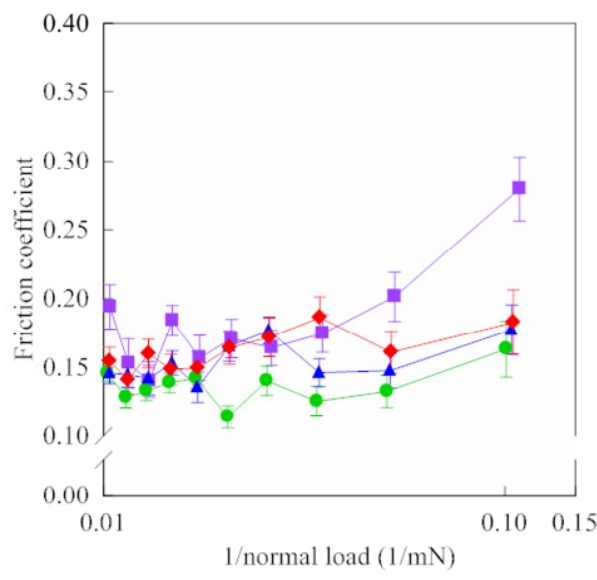

(b) $5 \mathrm{~mm} / \mathrm{sec}$

Fig. 6 Friction coefficients as a function of the reciprocal number of normal load of the bare Au surface, and HDT, $\mathrm{HDTOH}$, and EG3OMe SAMs. The average sliding speed is $1 \mathrm{~mm} / \mathrm{sec}$ (a) and $5 \mathrm{~mm} / \mathrm{sec}$ (b)

[22]. On the other hand, the HDTOH spectrum shows two peaks at $2850 \mathrm{~cm}^{-1}$ and $2920 \mathrm{~cm}^{-1}$, which were assigned to the $\mathrm{CH}_{2}$ symmetrical stretching mode and $\mathrm{CH}_{2}$ antisymmetric stretching mode, respectively. The EG3OMe spectrum shows two peaks at $2856 \mathrm{~cm}^{-1}$ and $2922 \mathrm{~cm}^{-1}$, which were assigned to the $\mathrm{CH}_{2}$ symmetrical stretching mode and $\mathrm{CH}_{2}$ antisymmetric stretching mode, respectively. The two peaks assigned to the $\mathrm{CH}_{2}$ stretching mode of the EG3OMe were broader than those of the HDT and HDTOH, indicating an amorphous and less-oriented conformation of the EG unit of the EG3OMe SAM [24]. The peaks assigned to the $\mathrm{CH}_{3}$ stretching mode were not observed with the EG3OMe. Only the perpendicular component of the dipole moment is active in IRRAS [25]. If the EG unit of EG3OMe are parallel to the surface, both the symmetric and asymmetric vibration of the $\mathrm{CH}_{3}$ group of the EG unit have components that are parallel to the surface and the peaks assigned to the $\mathrm{CH}_{3}$ stretching mode are not observed. Thus, it was considered that the EG unit of EG3OMe were parallel to the surface.

\subsection{Friction measurements}

Friction measurements were performed using our in-house developed pin-on-plate tribometer. Figure 6 shows the friction coefficient as a function of the reciprocal number of the normal load of the bare Au and the HDT, HDTOH, and EG3OMe SAMs. Errors estimated from the standard deviation are also shown in Fig. 6. In the low normal load region of sliding speeds of $1 \mathrm{~mm} / \mathrm{sec}$ (cf. Fig. 6(a)) and $5 \mathrm{~mm} / \mathrm{sec}$ (cf. Fig. 6(b)), the order of friction coefficients was as follows: EG3OM $<$ HDT $<$ HDTOH $<$ bare Au. We varied the sliding speed to examine the effect of the sliding speed upon the friction behavior. The effects of molecules upon the friction coefficients appeared in the low load region of a sliding speed of $1 \mathrm{~mm} / \mathrm{sec}$. On the other hand, in the low load region of a sliding speed of 5 $\mathrm{mm} / \mathrm{sec}$, the friction coefficients of the SAMs showed similar values. One possible reason for the effect of sliding speeds on the friction coefficients is that the different sliding speeds might change the molecular orientation which in turn led to a change in friction behavior. The friction coefficients of the HDT SAMs were lower than those of the HDTOH. Furthermore, the friction coefficients of the EG3OMe SAM were the lowest in the SAM surfaces used in this study. The HDT and HDTOH have the same number of alkyl unit and different end groups. Thus, the end group affected the lower friction coefficients of the HDT SAM more than that of the HDTOH SAM. On the other hand, the chain structures of the HDT and EG3OMe SAMs were different, indicating that the chain structure of the molecules also affected the friction properties. On the other hand, in the high normal load region, the friction coefficients were around 0.15 for all surfaces, indicating that the friction coefficients did not depend on the end groups or the chain structure of the SAMs. We think that the molecules were desorbed during the friction tests with high loads. Therefore, the friction coefficients of the SAM surfaces were similar to the bare Au surface.

3.5. The effects of molecules upon the friction properties

Now we discuss the relationship between the friction properties and molecules. In this study, we examined the effects of the end groups and chain structures. It has been reported that the thicknesses of the HDT, HDTOH, and EG3OMe SAMs were approximately $2 \mathrm{~nm}[22,26,27]$. The film thickness of the HDT and HDTOH SAMs was estimated using ellipsometry $[26,27]$ and that of the EG3OMe was estimated using the XPS results [22]. Figure 6 shows that the effects of molecules appeared only in the low normal load region. In our previous study [14], we analyzed normal octadecanethiol SAM surfaces after friction tests by XPS. In that study, 95\% of the molecules remained on surface after a sliding 
distance of $180 \mathrm{~m}$ under a load of $30 \mathrm{mN}$. The structure of the HDT and HDTOH molecules were similar to that of octadecanethiol, indicating that most parts of the molecules remained on the HDT and HDTOH SAMs at loads lower than $30 \mathrm{mN}$. In the case of the EG3OMe SAMs, we think that the SAMs remained after the friction tests because the friction coefficients were lower than those of the HDT and HDTOH SAMs and the bare $\mathrm{Au}$ surface under a load of $30 \mathrm{mN}$. On the other hand, in the high normal load region the molecules were desorbed during friction and the friction coefficients of the bare $\mathrm{Au}$ surface and the SAM surfaces were similar. Therefore, we can discuss the effects of molecules in the low normal load region.

First, we discuss the effects of the end groups on the friction in the low normal load region. The relationship between the friction and end groups has been reported by several researchers [16,28-30]. Houston et al. have investigated the relationship between the functional group of the SAMs and friction using interfacial force microscopy [28]. They prepared a surface and probe (tip) coated with alkanethiol SAMs and mercaptoalkanoic acid SAMs, which had different end groups of the methyl group and carboxyl group, respectively. They found that the combination of an alkanethiol SAM and a mercaptoalkanoic acid SAM exhibited a higher friction coefficient than that of the alkanethiol SAMs. Moreover, Green et al. have studied the effects on friction of several functional groups. These studies showed that the friction was expected to be larger on high surface energy materials [30]. Bhushan et al. have investigated adhesion, friction, and wear properties using five kinds of alkanethiol and biphenyl thiol SAMs with different end groups by AFM [16]. They reported that SAMs with end groups of the carboxyl group and hydroxyl group increased the adhesive force, leading to higher friction force. For these reasons, in our case, we supposed that the end group affected the higher friction coefficients of the HDTOH SAM more than the HDT SAM. The HDTOH SAM with the hydroxyl groups as the end group, which exhibited high surface energy, had a higher friction coefficient than the HDT SAM with the methyl group. The friction tests in this study were performed in air. Therefore, water content in the air should exist and might affect the results of the friction tests, indicating that the higher adhesion force that acted between the HDTOH SAM and the surface of the glass pin contributed to the high friction force. For the EG3OMe SAM, the contact angle was lower than that of the HDT SAM and higher than that of the HDTOH SAM. On the other hand, the friction coefficients of the EG3OMe SAM were lower than those of the HDT and HDTOH SAMs. This result indicated that wettability is not the only factor that affects the friction properties of SAMs with different chain structures and that the end groups affected the friction coefficients for the same chain structures.

Next, we discuss the effects of the chain structure of the molecules on the friction. The end groups of both the HDT and EG3OMe SAMs were the methyl group. The different friction coefficients of the HDT and EG3OMe SAMs were supposed to depend on the structures of their molecules. The structures of alkanethiol SAMs such as HDT SAMs on Au have been investigated by many researchers and these HDT SAMs had well-organized structures. The alkyl chain was sufficiently long and the intermolecular interaction of the HDT SAM was strong because the intermolecular interaction increased with longer alkyl chains. On the other hand, the EG3OMe SAMs have two units which are alkyl chains and oligo (ethylene glycol). We think this double-unit structure affects the tribological properties. In our previous study we reported that the double layer of the SAMs lowered their friction coefficients [15]. In that study, the topmost and lower layers acted as mobile and bound layers, respectively. The topmost layer was alkanethiol and the lower layer was mercaptoalkanoic acid. We think the double layers of the SAMs have similar structures of PFPE which is one of the conventional polymer lubricants with nano-order film thickness. It is well-known that the PFPE molecules in the lower layer that adsorb on the surface act as an immobile layer, behaving as a solid, and that those in the upper layer act as a mobile layer, like a liquid [17]. In the EG3OMe SAM, the effect seems to be similar. The alkyl chain units are thought to act as the bound layer because of the intermolecular interaction. On the other hand, the EG units are thought to have flexibility and to act as the mobile layer. The FT-IR data of the EG3OMe in the $\mathrm{CH}_{2}$ stretching region showed broad peaks. These results indicated an amorphous and less oriented conformation of the EG units of the EG3OMe SAM. This result also supported the flexibility of the EG units. We think that under friction the EG units and alkyl chains of the EG3OMe SAMs acted as mobile and bound layers, respectively, and contributed to lowering the friction.

Another possible reason for the low friction coefficients of EG3OMe SAM is the effect of water in air. The friction tests were performed in air and water existed between the pin and SAM surface. From the results of contact angle measurements in Table 2, the interaction between water and the end groups of SAMs are different. We think that the interaction between the water in the air and the end groups of the molecules affected the friction coefficients of the SAMs. Therefore, not only the molecular structure but also the appropriate interaction between water and molecules affected friction coefficients of SAMs. To examine the effect of molecular structure further, performing friction tests under varying levels of humidity could be a possible approach.

\section{Conclusions}

The tribological properties of organothiol SAMs on 
$\mathrm{Au}$ were investigated using a conventional pin-on-plate tribometer to clarify the effects of the structure and end groups of the molecules upon the frictional properties of the modified surfaces of the SAMs. The friction coefficients of the SAM surfaces were similar to those of the bare Au surface in the high normal load region. In the low normal load region, the order of the friction coefficients was as follows: EG3OM $<$ HDT $<\mathrm{HDTOH}$ $<$ bare Au. The HDT SAMs with the methyl group as an end group exhibited lower friction coefficients than the HDTOH SAMs with the hydroxyl group as an end group. The end groups affected the friction coefficients for the same chain structures. The friction coefficients of the EG3OMe SAM were lower than those of the HDT SAMs. We think that the HDT SAM had well-organized structures because of the intermolecular interaction of the $\mathrm{CH}_{2}$ groups of the alkyl chains. On the other hand, the EG3OMe SAM was probably more flexible than the HDT SAM because of its ethylene glycol chains, indicating that the chain structures as well as the end groups affected the friction coefficients of the SAMs.

\section{References}

[1] Ulman, A., "An Introduction to Ultrathin Organic Films From Langmuir-Blodgett to Self-Assembly," Academic Press, New York, 1991.

[2] Ulman, A, "Formation and Structure of Self-Assembled Monolayers," Chemical Reviews, 96, 4, 1996, 1533-1554.

[3] Ulman, A. (Ed), "Thin Films: Self-Assembled Monolayers of Thiols : 24," Academic Press, New York, 1998.

[4] Bhushan, B., Kulkarni, A. V., Koinkar, V. N., Boehm, M., Odoni, L., Martelet, C. and Belin, M. "Microtribological Characterization of Self-Assembled and Langmuir-Blodgett Monolayers by Atomic and Friction Force Microscopy," Langmuir, 11, 8, 1995, 3189-3198.

[5] DePalma, V. and Tillman, N., "Friction and Wear of Self-Assembled Trichlorosilane Monolayer Films on Silicon," Langmuir, 5, 3, 1989, 868-872.

[6] Cha, K. H. and Kim, D. E., "Investigation of the Tribological Behavior of Octadecyltrichlorosilane Deposited on Silicon," Wear, 251, 1-12, 2001, 1169-1176.

[7] Masuko, M., Miyamoto, H. and Suzuki, A., "Tribological Chracteristics of Self-Assembled Monolayer with Siloxane Bonding to Si Surface," Tribology International, 40, 10-12, 2007, 1587-1596.

[8] Srinivasan, U., Houston, M. R., Howe, R. T. and Maboudian, R., "Alkyltrichlorosilane-Based SelfAssembled Monolayer Films for Stiction Reduction in Silicon Micromachines," Journal of
Microelctromechanical Systems, 7, 2, 1998, 252-260.

[9] de Boer, M. P. and Mayer, T. M., "Tribology of MEMS," MRS Bulletin, 26, 4, 2001, 302-304.

[10] Satyanarayana, N. and Sinha, S. K., "Tribology of PFPE Overcoated Self-Assembled Monolayers Deposited on Si Surface," Journal of Physics D: Applied Physics, 38, 18, 2005, 3512-3522.

[11] Satyanarayana, N., Gosvami, N. N., Sinha, S. K. and Srinivasan, M. P., "Friction, Adhesion and Wear Durability of an Ultra-Thin Perfluoropolyether-Coated

3-glycidoxypropyltrimethoxy Silane Self-Assembled Monolayer on a Si Surface," Philosophical Magazine, 87, 22, 2007, 3209-3227.

[12] Lio, A., Charych, D. H. and Salmeron, M., "Comparative Atomic Force Microscopy Study of the Chain Length Dependence of Frictional Properties of Alkanethiols on Gold and Alkylsilanes on Mica," Journal of Physical Chemistry B, 101, 19, 1997, 3800-3805.

[13] Nakano, M., Ishida, T., Numata, T., Ando, Y. and Sasaki, S., "Alkyl Chain Length Effect on Tribological Behavior of Alkanethiol Self-Assembled Monolayers on Au," Japanese Journal of Applied Physics, 42, 7B, 2003, 4734-4738.

[14] Nakano, M., Ishida, T., Numata, T., Ando, Y. and Sasaki, S., "Tribological Behavior of Terphenyl Self-Assembled Monolayer Studied by a Pin-on-Plate Method and Friction Force Microscopy," Japanese Journal of Applied Physics, 43, 7B, 2004, 4619-4623.

[15] Nakano, M., Ishida, T., Numata, T., Ando, Y. and Sasaki, S., "Tribological Behavior of Self-Assembled Double Layer Measured by a Pin-on-Plate Method," Applied Surface Science, 242, 3-4, 2005, 287-294.

[16] Bhushan, B. and Liu, H., "Nanotribological Properties and Mechanisms of Alkylthiol and Biphenyl Thiol Self-Assembled Monolayers Studied by AFM," Physical Review B, 63, 24, 2001, 245412-1-245412-11.

[17] Khurshudov, A. and Waltman, R. J., "Tribology Challenges of Modern Magnetic Hard Disk Drives," Wear, 251, 1-12, 2001, 1124-1132.

[18] King, D. E., "Oxidation of Gold by Ultraviolet Light and Ozone at $25{ }^{\circ} \mathrm{C}$," Journal of Vacuum Science and Technology A, 13, 3, 1995, 1247-1253.

[19] Ron, H. and Rubinstein, I., "Alkanethiol Monolayers on Preoxidized Gold. Encapsulation of Gold Oxide under an Organic Monolayer," Langmuir, 10, 12, 1994, 4566-4573.

[20] Ishida, T., Tsuneda, S., Nishida, N., Hara, M., Sasabe, H. and Knoll, W., "Surface-Conditioning Effect of Gold Substrates on Octadecanethiol 
Self-Assembled Monolayer Growth," Langmuir, 13, 17, 1997, 4638-4643.

[21] Chatain, J. (Ed.), "Handbook of X-ray Photoelectron Spectroscopy," Perkin-Elmer Corporation, 1993.

[22] Harder, P., Grunze, M., Dahint, R., Whitesides, G. M. and Laibinis, P. E., "Molecular Conformation in Oligo(ethylene glycol)-Terminated Self -Assembled Monolayers on Gold and Silver Surfaces Determines Their Ability to Resist Protein Adsorption," Journal of Physical Chemistry B, 102, 2, 1998, 426-436.

[23] Li, L., Chen, S., Zheng, J., Ratner, B. D. and Jiang, S., "Protein Adsorption on Oligo(ethylene glycol) -Terminated Alkanethiolate Self -Assembled Monolayers: the Molecular Basis for Nonfouling Behavior,". Journal of Physical Chemistry B, 109, 7, 2005, 2934-2941.

[24] Herrwerth, S., Eck, W., Reinhardt, S. and Grunze, M., "Factors that Determine the Protein Resistance of Oligoether Self-Assembled Monolayers Internal Hydrophilicity, Terminal Hydrophilicity, and Lateral Packing Density," Journal of American Chemical Society, 125, 31, 2003, 9359-9366.

[25] Scheuing, D. R. (Ed), "Fourier Transform Infrared Spectroscopy in Colloid and Interface Science," American Chemical Society Press, Washington, DC, 1990, 149 .
[26] Bain, C. D., Troughton, E. B., Tao, Y.-T., Evall, J., Whitesides, G. M. and Nuzzo, R., "Formation of Monolayer Films by the Spontaneous Assembly of Organic Thiols from Solution onto Gold," Journal of American Chemical Society, 111, 1, 1989, 321-335.

[27] Wei, Y. and Latour, R. A., "Benchmark Experimental Data Set and Assessment of Adsorption Free Energy for Peptide-Surface Interactions," Langmuir, 25, 10, 2009, 5637-5646.

[28] Houston, J. E. and Kim, H. I, "Adhesion, Friction, and Mechanical Properties of Functionalized Alkanethiol Self-Assembled Monolayers," Accounts of Chemical Research, 35, 7, 2002, 547-553.

[29] Carpick, R. W. and Salmeron, M., "Scratching the Surface: Fundamental Investigations of Tribology with Atomic Force Microscopy," Chemical Review, 97, 4, 1997, 1163-1194.

[30] Green, J. -B. D., McDermott, M. T., Porter, M. D. and Siperko, L. M. "Nanometer-Scale Mapping of Chemically Distinct Domains at Well-Defined Organic Interfaces Using Frictional Force Microscopy," Journal of Physical Chemistry, 99, 27, 1995, 10960-10965. 